\title{
Adsorption Kinetics of Single-Stranded DNA on Functional Silica Surfaces and Its Influence Factors: An Evanescent-Wave Biosensor Study
}

\author{
Jun Wu, Hongliang Wang, Anna Zhu,* and Feng Long*(i) \\ School of Environment and Natural Resources, Renmin University of China, Beijing 10085, China
}

Supporting Information

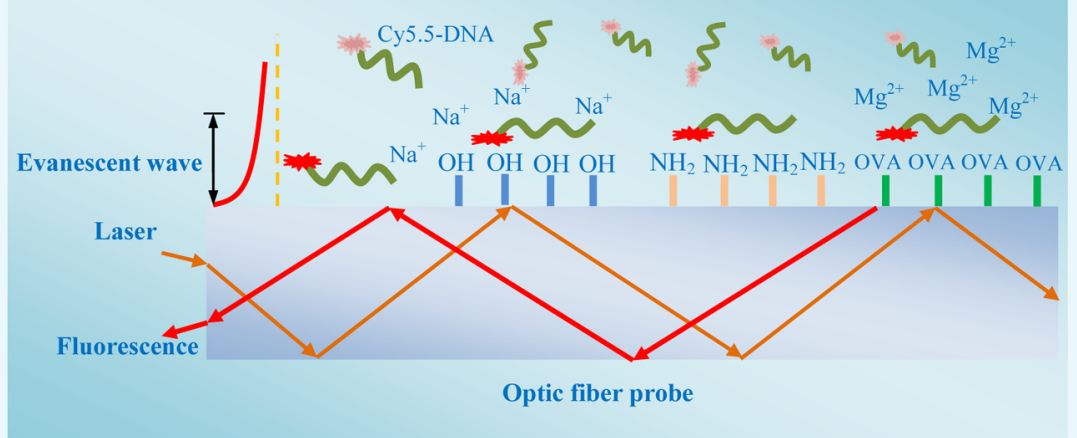

\begin{abstract}
Thorough understandings on the real-time kinetics involved in DNA adsorption on a solid surface is essential in various fields, such as in DNA hybridization studies, DNA extraction and purification, DNA-based biosensing, and gene-based medicine discovery. Herein, the real-time properties of single-stranded DNA (ssDNA) adsorption onto functional silica surfaces under various conditions were investigated using an evanescent wave optical biosensing platform. Results demonstrated that the driving force and adsorption mechanism of DNA were closely related to the kind of functional groups on the silica surfaces. The main driving forces of DNA adsorption onto hydroxyl- and protein-modified solid surfaces were the hydrophobic interaction, hydrogen bonding, and the interaction between DNA phosphate and functional groups on the silica surface, which strengthened with increased ionic strength. However, the electrostatic attraction between the negative charge of DNA and positive charge of the amino silica surface was likely the most important factor influencing DNA adsorption onto the amino surface. This influence can be reduced by increasing the ionic strength. Although low-ionic-strength $\mathrm{Mg}^{2+}$ provided a greater adsorption efficiency than high-ionic-strength $\mathrm{Na}^{+}$, the balance of ssDNA adsorption onto hydroxyl- and ovalbumin (OVA)-modified silica surfaces was achieved faster in the presence of $\mathrm{Na}^{+}$than in the presence of $\mathrm{Mg}^{2+}$. DNA adsorption was also influenced significantly by $\mathrm{pH}$, and the hydroxyl- and OVA-modified surfaces exhibited the strongest adsorption at $\mathrm{pH}$ 3.0, whereas DNA adsorption onto the amino surface increased with increased $\mathrm{pH}$. DNA adsorption onto various functional surfaces could be perfectly fitted by second-order Langmuir models, indicating that the process was a single-molecular-layer adsorption.
\end{abstract}

\section{INTRODUCTION}

The kinetics of DNA adsorption onto various solid substrates has attracted great scientific attention because of its widespread bioapplications, such as nucleic acid isolation and purification, ${ }^{1,2}$ clinical genetic analysis, ${ }^{3,4}$ gene delivery, 5,6 and biosensor design. ${ }^{7,8}$ Silica (i.e., amorphous silicon dioxide, $\mathrm{SiO}_{2}$ ) is an ideal substrate for DNA adsorption because of the material's advantages such as smooth surface, stable chemical properties, low toxicity, cost-effectiveness, and easy application in micro/nanoscale devices. ${ }^{9,10}$ The driving forces of DNA adsorption onto a silica surface include hydrophobic interactions, electrostatic interaction, and hydrogen bonding between DNA and the silicon dioxide surface. ${ }^{4,9,10}$ The interaction between DNA phosphate and silanol groups on the silica surface was also regarded as a principle mechanism for DNA adsorption. ${ }^{10}$ Although electrostatic repulsion exists between negatively charged DNA and a negatively charged silica surface above its isoelectric point (IEP), the phosphate silanol and hydrophobic interactions are sufficiently strong to overcome DNA/silica electrostatic repulsion, thus leading to DNA binding to the silica surface. Several investigators proposed that DNA adsorption occurs through hydrogen bonding between unwound nucleotides and the silica surface. ${ }^{4,10-12}$ By changing solution $\mathrm{pH}$, DNA can be adsorbed when the surface is positively charged and desorbed almost completely when the surface is tuned to be negatively charged. ${ }^{10-12}$ Cations, such as sodium $\left(\mathrm{Na}^{+}\right)$and magnesium $\left(\mathrm{Mg}^{2+}\right)$ ions, can be added to neutralize the negative charge for

Received: December 25, 2017

Accepted: February 20, 2018

Published: May 25, 2018 


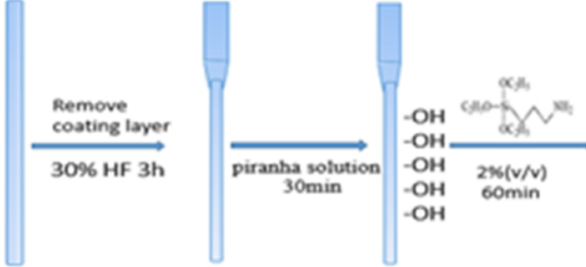

Fiber A

Fiber B

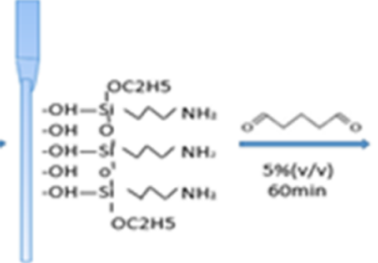

Fiber C

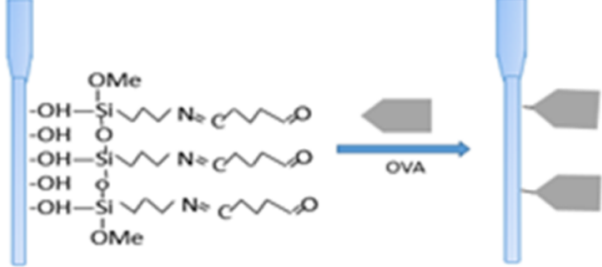

Fiber D

Figure 1. Preparation of functional optic fiber probes. Fiber A, naked etched optic fiber probe; fiber B, hydroxyl optic fiber probe; fiber C, amino optic fiber probe; and fiber D, OVA-modified optic fiber probe.

shielding electrostatic interaction and compressing the electrostatic double layer surrounding the DNA and the silica surface, which facilitates DNA adsorption onto silicon dioxide.

To date, DNA adsorption onto an acid-treated silica surface have been widely studied. Acid treatment can hydrolyze the silica surface and increase its silanol group concentration. As such, the treatment adds benefits to DNA adsorption. ${ }^{10}$ The physical or chemical modification of a silica surface is another approach to effectively adsorb DNA. For example, the silica surface modified with amino groups can increase the DNA adsorption amount by introducing direct electrostatic interactions. By contrast, DNA desorption is limited because of the lack of repulsive force. DNA could also be adsorbed effectively onto the nanoparticle surface modified by protein as a natural ampholyte with specified IEP. Meanwhile, the amount of DNA that binds to a silica surface depends on solution $\mathrm{pH},{ }^{12-14}$ ionic type, and strength, ${ }^{4,10-14}$ as well as DNA conformation. ${ }^{14,15}$ Numerous techniques, including equilibrium bulk depletion assay technologies, evanescent wave-induced fluorescent spectroscopy, ${ }^{16}$ atomic force microscopy, ${ }^{17}$ microscopy using intercalating fluorescent dyes, ${ }^{17,18}$ and dual polarization interferometry, ${ }^{19,20}$ have been employed to investigate the adsorption mechanism and enumerate the attractive forces between DNA and the silica surface. However, most of these techniques do not allow real-time monitoring of DNA adsorption kinetics. As a result, some key information is lost. $^{12}$ Recently, a quartz crystal microbalance with dissipation monitoring was used to measure the kinetics of DNA adsorption onto a piezoelectric quartz crystal. ${ }^{12}$

In this study, we investigated the adsorption kinetics of single-stranded DNA (ssDNA) onto a silicon dioxide (quartz) optic fiber probe by monitoring the real-time fluorescence changes using an evanescent wave all-fiber optical biosensor (EWAB). Previous studies suggested that both double-stranded DNA (dsDNA) and ssDNA can be absorbed by the silicon dioxide surface. $^{21}$ For analytical and biomedical applications, attaching ssDNA with well-defined sequences, such as aptamers, is more desirable. The ssDNA can bind to silicon dioxide more strongly than dsDNA when solution $\mathrm{pH}$ is above the IEP of silicon dioxide up to neutral $\mathrm{pH}^{13,18}$ This condition increases the opportunity for hydrophobic interaction between the unpaired bases of ssDNA and the silicon dioxide surface. $^{13,18}$ Herein, a $20 \mathrm{bp}$ random ssDNA sequence was regarded as a model for investigating DNA adsorption kinetics. Several functional optic fiber probes, including bare, hydroxyl, amino, and protein-modified optic fibers, were prepared to investigate ssDNA adsorption kinetics. The effects of $\mathrm{pH}$ and ionic species and strength on ssDNA adsorption onto the silicon dioxide surface were studied. To our best knowledge, few studies have compared the DNA adsorption kinetics onto various functional surfaces. Our experiments confirmed some previously proposed adsorption mechanisms of ssDNA onto the solid surface. Moreover, our results also provided additional important ssDNA adsorption kinetics information by real-time fluorescence trace. These results help us increase understanding of the basic principles underlying the interactions between solid surfaces and nucleic acids.

\section{RESULTS}

Characteristics of Functional Optic Fiber Probes. Starting from an etched optic fiber probe, we assembled a covalently bonded molecular monolayer through a multistep chemical process (Figure 1). ${ }^{21,22}$

To characterize the functional optic fiber probes, the results of X-ray photoelectron spectroscopy (XPS) were shown in Table 1 and Figure S2. After etched by HF and washed by

Table 1. XPS Results of Functional Optic Fiber Probes

\begin{tabular}{ccrrrl}
$\begin{array}{c}\text { functional } \\
\text { optic fiber } \\
\text { probe }\end{array}$ & $\begin{array}{c}\text { percent of } \\
\text { element/binding } \\
\text { energy }\end{array}$ & \multicolumn{1}{c}{$\mathrm{C}_{1 \mathrm{~s}}$} & \multicolumn{1}{c}{$\mathrm{O}_{1 \mathrm{~s}}$} & \multicolumn{1}{c}{$\mathrm{Si}_{2 \mathrm{p}}$} & \multicolumn{1}{c}{$\mathrm{N}_{1 \mathrm{~s}}$} \\
fiber A & atom/\% & 15.72 & 58.04 & 26.24 & 0 \\
& B.E./eV & 284.71 & 530.75 & 101.56 & $\mathrm{NA}^{a}$ \\
fiber B & atom/\% & 16.12 & 57.73 & 26.17 & 0 \\
& B.E./eV & 284.82 & 530.87 & 101.62 & $\mathrm{NA}^{a}$ \\
fiber C & atom/\% & 26.17 & 49.59 & 23.26 & 0.46 \\
& B.E./eV & 284.70 & 530.80 & 101.60 & 398.70 \\
fiber D & atom/\% & 51.73 & 30.07 & 15.79 & 2.25 \\
& B.E./eV & 284.67 & 530.78 & 101.53 & 398.82 \\
${ }^{a}$ NA: no absorption was detected. & & & \\
\hline
\end{tabular}

water, the tapered probe surface mainly contained carbon, oxygen, and silicon. Then, the elemental composition is basically not changed after acid treatment. However, after 3aminopropyltriethoxysilane (APTS) was coupled onto the fiber optic probe, the XPS spectra showed nitrogen (1.24\%) because the APTS contained nitrogen, which showed that the APTS successfully cross-linked to the optic fiber probe through conjugation of the hydroxyl case on the probe surface and the silane group of APTS. Finally, ovalbumin (OVA) was covalently conjugated onto the probe surface using the glutaraldehyde solution. The amount of nitrogen on the probe surface increased from 0.46 to $2.25 \%$ because of protein coupling.

Hydrophobic/hydrophilic property is essential in DNA adsorption. Hence, the hydrophobic/hydrophilic properties of the silica-based surface were characterized after modification by different functional groups. The optic fiber probe is cylindrical; hence, its contact angle cannot be obtained. The contact angle of the silicon dioxide plate was determined using the abovementioned methods. In this case, the plate functioned as the 


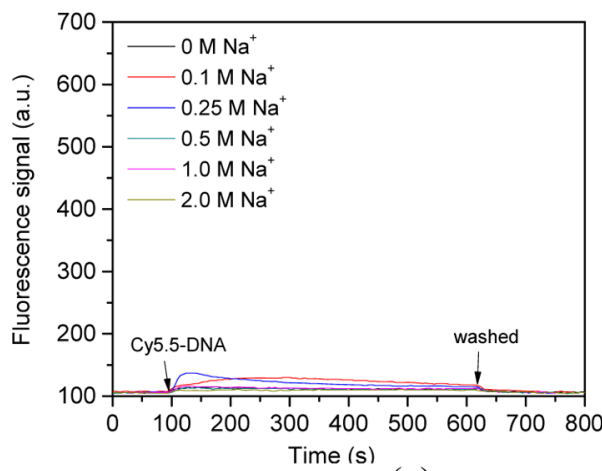

(a)

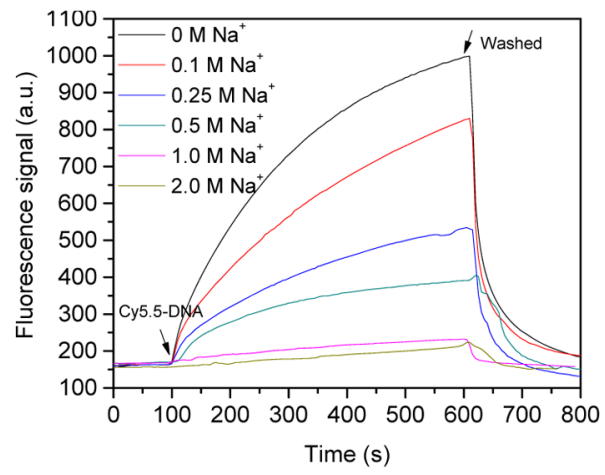

(c)

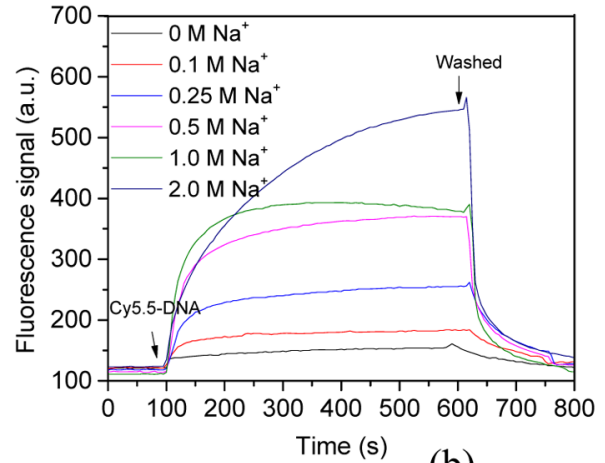

(b)

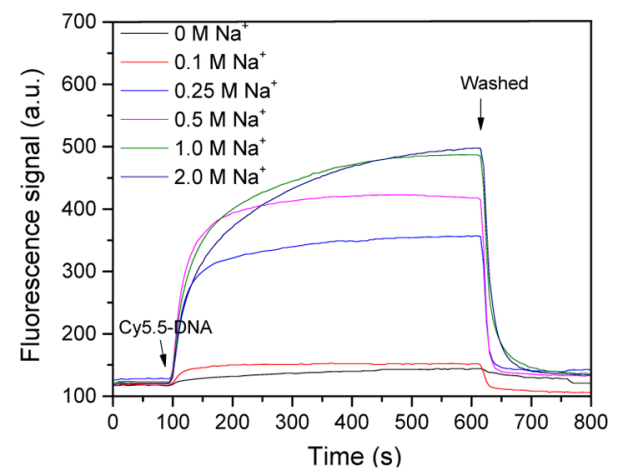

(d)

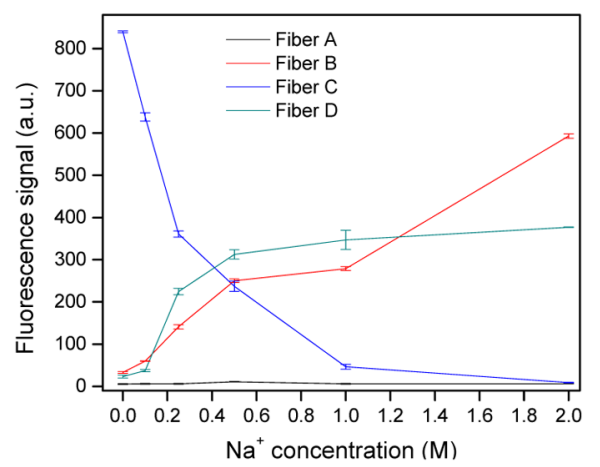

(e)

Figure 2. Adsorption kinetics of ssDNA onto functional optic fiber probes under different $\mathrm{Na}^{+}$concentrations. (a) Fiber A; (b) fiber B with hydroxyl groups; (c) fiber $\mathrm{C}$ with amino groups; (d) fiber D covering with OVA; and (e) fluorescence signal of different functional optic fiber probes at equilibrium adsorption. ssDNA concentration was $20 \mathrm{nM}$. The error bars represent standard deviation from three measurements.

optic fiber probe. The bare plate was highly hydrophilic $\left(<10^{\circ}\right)$ as in previous research. ${ }^{25}$ After the plate was treated by acid, the hydroxyl silicon dioxide surface attained a large contact angle $\left(24.8^{\circ}\right)$ (Figure S3). Specially, the hydrophobic property of the silica-based surface increased when hydroxylated. Furthermore, after modification by APTS, the amino group was conjugated on the silica surface, and the silica surface became the most hydrophobic at a contact angle of $72.3^{\circ}$. Therefore, the amino fiber $\mathrm{C}$ was likely hydrophobic after covalent bonding with APTS. Finally, the OVA was covalently bound to the amino silica surface at a contact angle of $25.3^{\circ}$. The OVA-modified surface became hydrophobic as did the hydroxyl surface owing to protein properties.

Kinetics of ssDNA Adsorption onto Functional Optic Fiber Probes under Monovalent Cation. To obtain information about the adsorption kinetics in real time and to investigate the adsorption behavior of ssDNA adsorbed onto the surface, we studied ssDNA adsorption to the optic fiber probe surface using EWAB under different ionic species and strengths. To study the effect of ionic strength on ssDNA adsorption onto optic fiber probes, we used the ultrapure water but not the phosphate-buffered saline (PBS) to prepare ssDNA solution. In this section, different concentrations of $\mathrm{Na}^{+}$were added to the ssDNA solution and were introduced into the optofluidic cell. Concurrently, the real-time fluorescence signal was recorded by EWAB.

In these experiments, we found that the functionalization of the probe surface obviously affected the adsorption kinetics and the amount of DNA adsorbed. Hence, different shaped fluorescence response curves were obtained. First, scarce DNA amount was adsorbed on the optic fiber probe surface unless the etched optic fiber probes were functionally pretreated (Figure 2a), as in previous reports. ${ }^{25}$ When Cy5.5labeled DNA was delivered over the probe surface, a weak fluorescence signal was detected. Then, balance was rapidly reached once the Cy5.5-labeled DNA solution filled the 


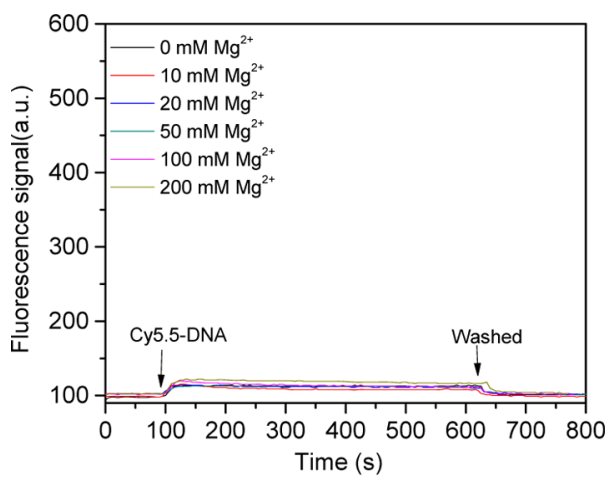

(a)

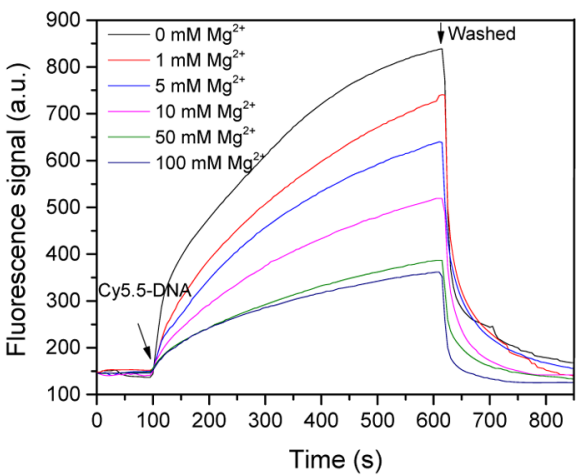

(c)

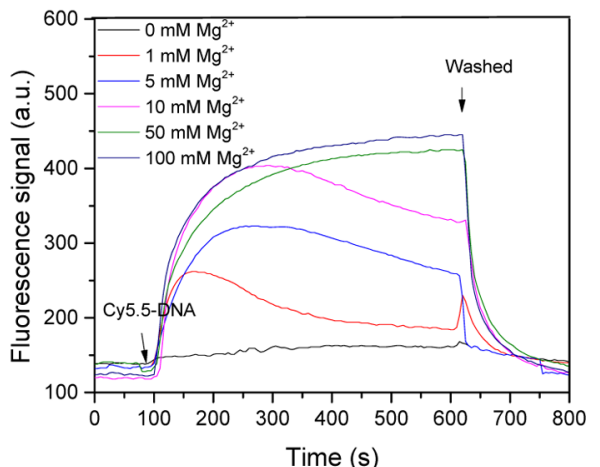

(b)

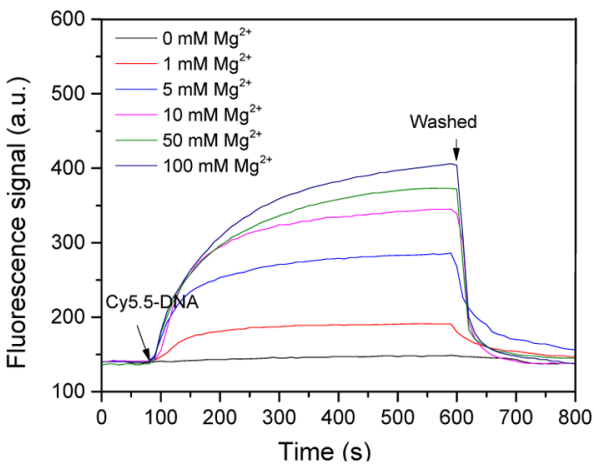

(d)

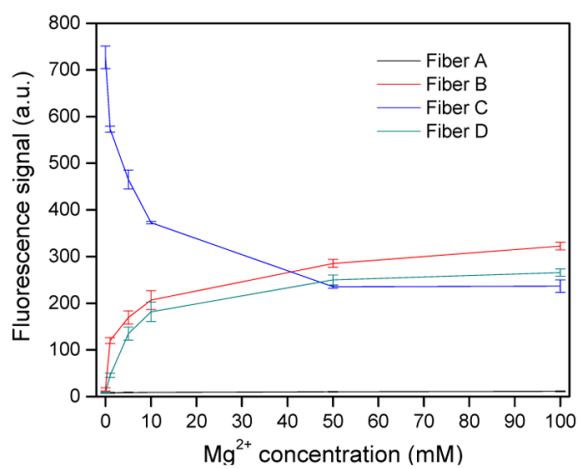

(e)

Figure 3. Kinetics of ssDNA adsorption onto functional optic fiber probes at different $\mathrm{Mg}^{2+}$ concentrations. (a) Fiber A; (b) fiber B with hydroxyl groups; (c) fiber C with amino groups; (d) fiber D covered with OVA; and (e) fluorescence signal of different functional optic fiber probes at equilibrium adsorption, in which the ssDNA concentration was $20 \mathrm{nM}$. The error bars represent standard deviation from three measurements.

optofluidic cell. No adsorption process was observed, and the fluorescence signal rapidly reached the baseline after washing with water. In general, the DNA-silica interaction is electrostatically unfavorable because DNA and the silica surface are both negatively charged under most experimental conditions. ${ }^{25}$ Adding electrolytes shields electrostatic repulsion. However, despite the high $\mathrm{Na}^{+}$concentration employed, fiber A only exhibited a slight response to the addition of the Cy5.5-labeled DNA (Figure 2e). Thus, the electrostatic repulsion was not the response in this case. Silica surfaces can display one or two $\mathrm{OH}$ groups per surface silica atom (called isolated or geminal hydroxyls) besides bridging ethereal oxygens between silica surface atoms. ${ }^{26}$ Even so, perfect $\mathrm{SiO}_{2}$ (quartz) exists mainly in the bridging ethereal oxygen conformation and possesses no free surface hydroxyl groups, thus producing a highly hydrophilic surface (contact angle $<10^{\circ}$ ). The high hydrophilicity of the probe is likely a main factor why DNA did not adsorb onto the probe surface. Furthermore, the effective distance of the evanescent wave was less than $100 \mathrm{~nm}$ and only Cy5.5 in this range can be excited. Thus, we can assume that the fluorescence signal to be detected may originate from the free Cy5.5-labeled DNA in solution near the probe surface. This notion suggests that the free fluorescence-labeled DNA only slightly contributes to the detected fluorescence signal detected by EWAB.

When the optic fiber probe was treated using piranha solution, a typical adsorption process was observed when Cy5.5-labeled ssDNA was introduced over the probe surface (Figure $2 \mathrm{~b}$ ). Acid treatment of silica hydrolyzes the surface and increases the silanol groups of the probe surface. The amount of ssDNA adsorbed initially increased rapidly in the presence of $\mathrm{Na}^{+}$(Figure 2b). With continuous adsorption, the adsorbed ssDNA increased less rapidly for about $100 \mathrm{~s}$ until a plateau was reached. The adsorption of ssDNA onto the probe surface may 
be attributed to the hydrophobic interactions, hydrogen bonding, and the interaction between DNA phosphate and the probe surface silanol groups. Moreover, the fluorescence intensified with increased $\mathrm{Na}^{+}$concentration (Figure $2 \mathrm{~b}, \mathrm{e}$ ). This phenomenon may be ascribed to the shielding against electrostatic repulsion and compression of the electrostatic double layer surrounding the DNA and the silica surface derived from electrolyte addition. Then, these effects facilitate DNA adsorption onto silica. Previous studies demonstrated that hydrated $\mathrm{Na}^{+}$can interact with the oxygen atoms of DNA phosphate groups directly or through hydrogen bonds with metal-coordinated water molecules. ${ }^{27,28}$ The electrostatic neutralization of cations by the DNA phosphate backbone could reduce the thickness of the effective DNA diameter and compact the DNA onto the probe surface. This occurrence increases fluorescence-labeled DNA adsorption onto the probe surface and consequently intensifies the detected fluorescence signal.

Then, the probe surfaces were further functionalized by aminosilane-coupling reagents. The amount of ssDNA adsorbed initially increased because of the strong electrostatic attraction between the negatively charged DNA phosphate groups and the positively charged amino groups on the amino probe surface (Figure 2c), especially at low cation concentrations. The hydrophobic interaction between the DNA and the amino probe surface may also serve as another important reason. The adsorbed ssDNA gradually increased, and a plateau was reached at longer time than that with the hydroxyl probes. Moreover, the fluorescence signal change differs from that of the hydroxyl probes (Figure 2c). The adsorption capacity decreased with increased $\mathrm{Na}^{+}$concentration because the fluorescence signal decreased with increased cation strength (Figure 2e). When $\mathrm{Na}^{+}$was $2.0 \mathrm{M}$, few DNA adsorbed onto the probe surface, which was different from previous results. ${ }^{25}$ This appearance may be attributed to the use of different buffer solutions, and our solution only contained $\mathrm{Na}^{+}$. Hydrated $\mathrm{Na}^{+}$ can interact with the oxygen atoms of DNA phosphate groups; ${ }^{27}$ hence, the electrostatic neutralization of cations with the DNA phosphate backbone could reduce the electrostatic interaction between DNA and the silica surface with increased $\mathrm{Na}^{+}$concentration. Therefore, DNA may even be positively charged at high $\mathrm{Na}^{+}$concentration, which resulted in the electrostatic repulsion between DNA and the positively charged amino probe surface. Thus, a low amount of DNA adsorbed onto the probes surface.

When the probe surfaces were covalently coupled by OVA, the resultant DNA adsorption performance was similar to that of the hydroxyl probes. The amount of ssDNA adsorbed initially increased when Cy5.5-labeled DNA was introduced into the sample cell. With continuous adsorption, the adsorbed ssDNA increased less rapidly for about $50 \mathrm{~s}$ until a plateau was reached. Moreover, the adsorption capacity increased with cation strength linearly at low cation concentration and reached a plateau when the $\mathrm{Na}^{+}$concentration exceeded $0.5 \mathrm{M}$. The OVA-modified probes achieved a slightly higher adsorbed amount of DNA than that by the hydroxyl probes in the presence of $\mathrm{Na}^{+}$. As a result, both probes became negatively charged with similar contact angles. However, the OVAmodified probes were more sensitive to cation strength than hydroxyl probes because the adsorption slope of the former was higher than that of the latter (Figure 2e). When the $\mathrm{Na}^{+}$ concentration was $0.25 \mathrm{M}$, the fluorescence signal was about $70 \%$ of the maximum fluorescence signal value.
Kinetics of ssDNA Adsorption onto Functional Optic Fiber Probes under Divalent Cation. In this section, the effect of divalent cation strength $\left(\mathrm{Mg}^{2+}\right)$ on DNA adsorption onto the probe surface was investigated. Similar to observations under the use of monovalent cation, very small DNA amount adsorbed onto the bare optic fiber surface when Cy5.5-labeled DNA was delivered over the probe surface (Figure 3a). Moreover, regardless of the increase in $\mathrm{Mg}^{2+}$ concentration, fiber A had shown a weak response for the Cy5.5-labeled DNA (Figure 3e).

When Cy5.5-labeled DNA was introduced over the hydroxyl probe surface, a typical adsorption process was observed in the presence of $\mathrm{Mg}^{2+}$ (Figure 3b). However, although the DNA adsorption amount increased over time when the $\mathrm{Mg}^{2+}$ concentration exceeded $50 \mathrm{mM}$, the adsorption curves obviously differed as the $\mathrm{Mg}^{2+}$ concentration varied from 10 to $50 \mathrm{mM}$. In the latter case, the fluorescence signal detected by EWAB initially increased rapidly and then decreased with continuous adsorption. This trend is difficult to be observed unless a real-time adsorption kinetic curve is recorded. According to previous studies, DNA and divalent cation could form DNA/cation complexes and be adsorbed onto the solid surface. ${ }^{29}$ At low ion concentration, adsorbed DNA film is viscoelastic. ${ }^{29}$ Specially, DNA initially adsorbs in a relatively flat conformation and interacts with the silica surface through many binding sites. As the DNA surface coverage increases, the adsorbed DNA rearranges, extending further into solution while interacting with the silica surface through fewer binding contacts, thus leading to a decrease in the fluorescence signal. At high ion concentration, the adsorbed DNA layer is rigid, the adsorbed DNA did not rearrange, and the detected fluorescence signal increases with the process of DNA adsorption. However, these results appeared only when the hydroxyl probe was used.

When Cy5.5-labeled ssDNA was introduced over the amino probe surface, the amount of ssDNA adsorbed initially increased, especially in the absence of or in low $\mathrm{Mg}^{2+}$ concentrations (Figure 3c). These effects should contribute to the strong electrostatic and hydrophobic interaction between the negatively charged DNA phosphate groups and the positively charged amino groups on the functional probe. With continuous adsorption, the adsorbed ssDNA increased less rapidly and a plateau was reached. Adsorption capacity decreased following the increase of $\mathrm{Mg}^{2+}$ concentration as that observed in the presence of $\mathrm{Na}^{+}$. This effect was due to the decreased fluorescence signal with increased cation strength (Figure $3 \mathrm{e}$ ). DNA and $\mathrm{Mg}^{2+}$ can form DNA/ $\mathrm{Mg}^{2+}$ complexes; hence, the electrostatic neutralization of cations with DNA could reduce electrostatic attraction between the DNA and silica surface with increased $\mathrm{Mg}^{2+}$ concentration. This phenomenon results in diminished DNA adsorption onto the silica surface and a weak fluorescence signal. We assumed that with increased cation strength, the charges of the amino surfaces and DNA phosphate groups were screened by the high salt concentration, leading to decreased DNA adsorption onto the probe surface. However, part of the DNA may still adsorb onto the probe surface, unlike that in presence of $\mathrm{Na}^{+}$. The amount of DNA adsorption did not decrease with increased $\mathrm{Mg}^{2+}$ concentration even when the $\mathrm{Mg}^{2+}$ concentration exceeded $50 \mathrm{mM}$. This observation showed that the interaction of DNA with $\mathrm{Mg}^{2+}$ differed from that with $\mathrm{Na}^{+}$. DNA/ $\mathrm{Mg}^{2+}$ complexes only partially neutralized the DNA charges and did not induce a conversion to positive charge. 


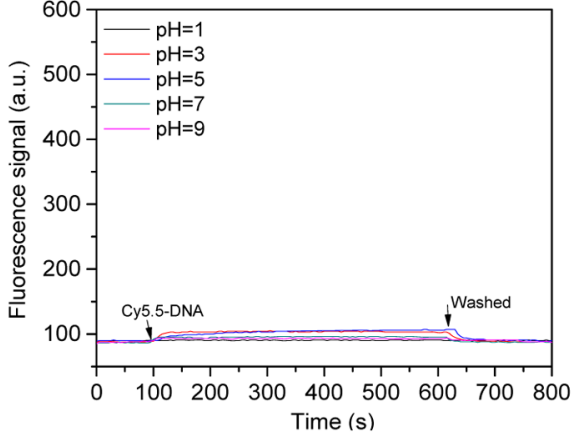

(a)

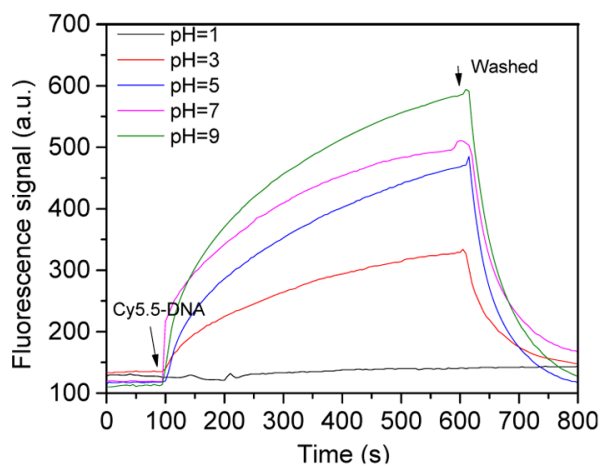

(c)

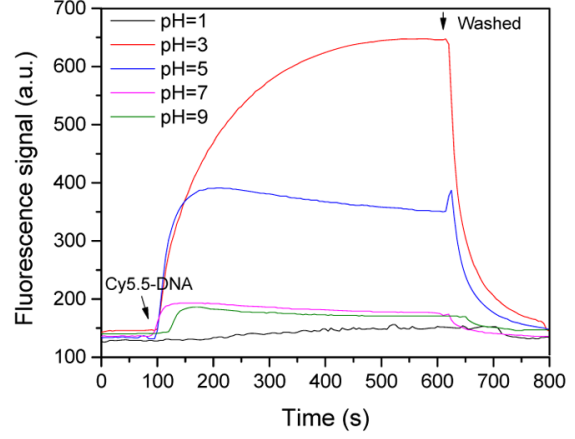

(b)

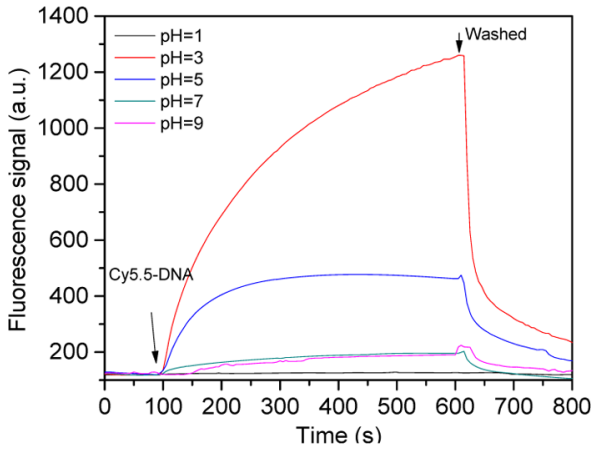

(d)

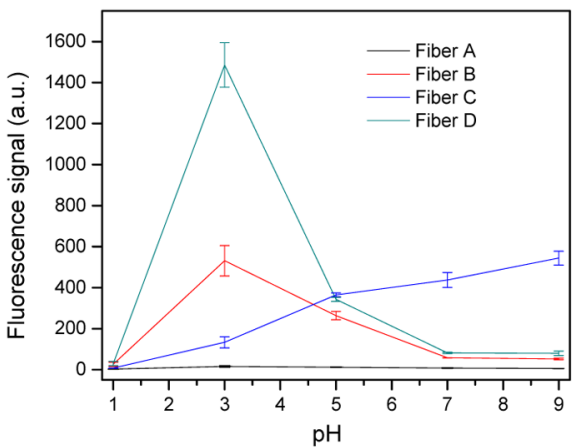

(e)

Figure 4. Kinetics of ssDNA adsorption onto functional optic fiber probes under different $\mathrm{pH}$ values. (a) Fiber A; (b) fiber B with hydroxyl groups; (c) fiber $\mathrm{C}$ with amino groups; (d) fiber D with OVA protein; and (e) fluorescence signal of different functional optic fiber probes at equilibrium adsorption, in which the ssDNA concentration was $20 \mathrm{nM}$. The error bars represent standard deviation from three measurements.

The amount of ssDNA adsorbed initially increased when Cy5.5-labeled ssDNA was introduced over the OVA-modified fiber optic probe (Figure 3d) similar to that in the hydroxyl probes. The efficiency of DNA adsorption onto the probe surface increased over time. The absorption efficiency augmented with raised $\mathrm{Mg}^{2+}$ concentration because of the suppressed electrostatic attraction between the positively charged particle surface and the DNA phosphate groups. The OVA-modified probes attained a slightly lower adsorbed DNA amount than that obtained through hydroxylation probes. Moreover, the adsorption capacity linearly increased with cation strength at low cation concentration and reached a plateau when the $\mathrm{Mg}^{2+}$ concentration exceeded $50 \mathrm{mM}$.

Kinetics of ssDNA Adsorption under Different $\mathrm{pH}$ Conditions. Besides cation species, DNA adsorption and the corresponding conformation are also influenced significantly by $\mathrm{pH}^{10,14}$ The behaviors of DNA adsorption onto various functional optic fiber probes under different $\mathrm{pH}$ values $(1,3,5$,
7, and 9) were also investigated (Figure 4). For the bare optic fiber probe, very small DNA amount adsorbed onto the probe surfaces at all $\mathrm{pH}$ conditions (Figure 4a). Hence, DNA adsorption onto bare optic fiber probe was also not affected by $\mathrm{pH}$.

For the acid-treatment probe, the amount of DNA adsorbed increased over time at $\mathrm{pH}<3.0$ (Figure $4 \mathrm{~b}$ ). However, the amount of DNA adsorption initially increased over time and then slightly decreased at $\mathrm{pH}>3.0$. This observation may be related to the interaction strength between the DNA and the silica surface similar to that in the presence of $\mathrm{Mg}^{2+}$. The DNA adsorption efficiency onto the probe surface was maximum at $\mathrm{pH} 3.0$ and decreased sharply when solution $\mathrm{pH}$ was less than 3.0 or higher than 5.0 (Figure $4 \mathrm{~b}, \mathrm{e}$ ). These results were qualitatively similar to those in previous studies. ${ }^{12,30}$ Increasing $\mathrm{pH}$ increases the electrostatic repulsion between the DNA and silica, which is likely a major factor contributing to the decreased quantity of adsorbed DNA. 


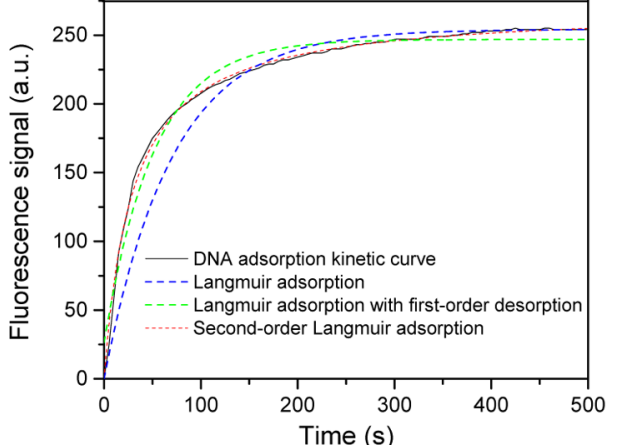

(a)

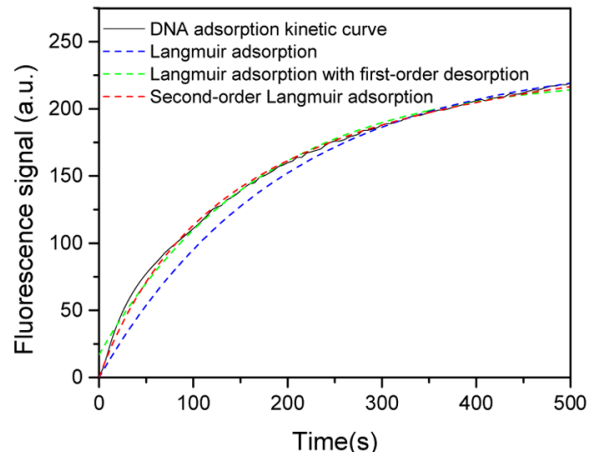

(c)

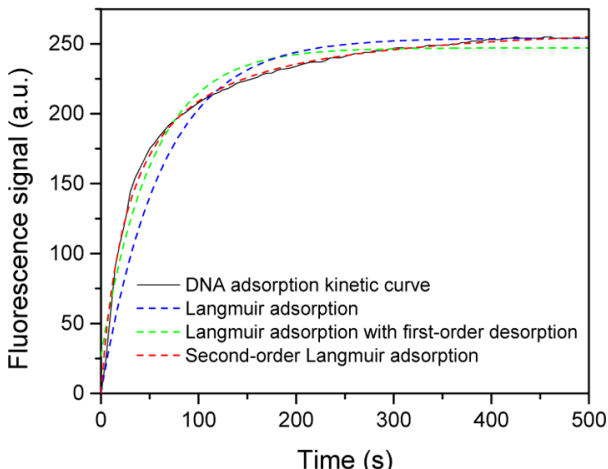

(e)

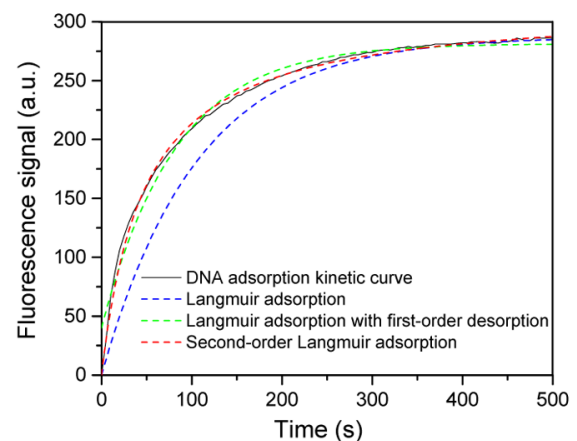

(b)

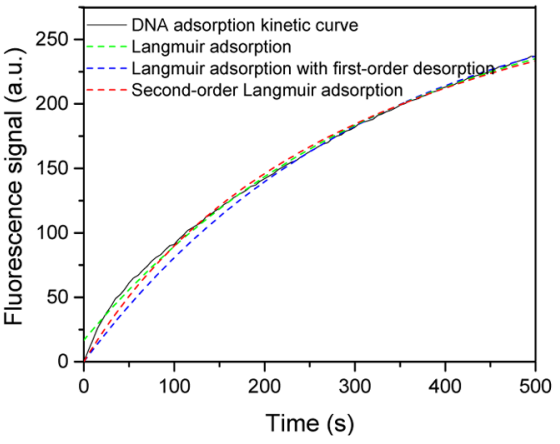

(d)

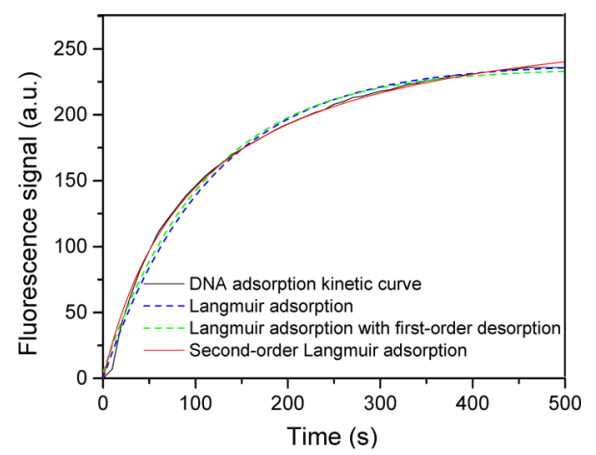

(f)

Figure 5. Kinetic curves of ssDNA adsorption onto functional optic fiber probes fitted by various models. (a) ssDNA (20 nM) adsorption onto hydroxyl fiber B in $0.5 \mathrm{M} \mathrm{Na}^{+}$solution; (b) $20 \mathrm{nM}$ ssDNA adsorption onto hydroxyl fiber B in $100 \mathrm{mM} \mathrm{Mg}^{2+}$ solution; (c) $20 \mathrm{nM}$ ssDNA adsorption onto amino fiber $\mathrm{C}$ in $0.5 \mathrm{M} \mathrm{Na}^{+}$solution; (d) $20 \mathrm{nM}$ ssDNA adsorption onto amino fiber $\mathrm{C}$ in $0.5 \mathrm{M} \mathrm{Na}^{+}$solution; (e) $20 \mathrm{nM}$ ssDNA adsorption onto OVA-modified fiber D in $0.5 \mathrm{M} \mathrm{Na}^{+}$solution; and (f) $20 \mathrm{nM}$ ssDNA adsorption onto OVA-modified fiber $\mathrm{D}$ in $0.5 \mathrm{M} \mathrm{Mg}^{2+}$ solution.

$\mathrm{pH}$ effects on DNA adsorption onto the aminosilanemodified optic fiber probe were observed between $\mathrm{pH} 1$ and 9 (Figure 4c). Efficient DNA adsorption increased with increased $\mathrm{pH}$, and the maximum DNA adsorption occurred at $\mathrm{pH}$ 9.0, indicating the correlation of DNA adsorption with surface positive charges. Amino groups present at $\mathrm{pH} 9.0$ are expected to be deprotonated because the $\mathrm{p} K_{\mathrm{a}}$ of aminosilane is estimated at $9-10 .^{14,29}$ By contrast, the protonation of amino groups on the aminosilane-modified probes is expected at $\mathrm{pH}$ less than 9. Generally, low $\mathrm{pH}$ and high salt concentration are beneficial to DNA adsorption. However, DNA adsorption at different $\mathrm{pH}$ values and salt concentrations depended on the existing functional group and hydrophobic property of the solid surface in our experimental results.

The kinetics of ssDNA adsorption onto the OVA-modified probes under different $\mathrm{pH}$ was also investigated. The DNA absorption efficiency onto the probe surface was highest at $\mathrm{pH}$
3.0 and decreased sharply to $20 \%$ when the solution $\mathrm{pH}$ was risen to 5.0 (Figure $4 \mathrm{~d}$ ). At $\mathrm{pH}>5.0$, almost no DNA absorption onto the probe surface was detected. The absorption efficiency decreased with increased $\mathrm{pH}$ because of the suppressed electrostatic attraction between the positively charged particle surface and the phosphate group of DNA $\left(\mathrm{p} K_{1}\right.$ = 2.1). At $\mathrm{pH}>5.0$, DNA absorption was blocked almost completely by the electrostatic repulsion because both DNA and the probe surface were negatively charged in this $\mathrm{pH}$ region. The OVA-modified probe attained a higher DNA adsorption amount than the hydroxyl probe because the former probe was positively charged at $\mathrm{pH}$ lower than the IEP and negatively charged at $\mathrm{pH}$ higher than IEP. Meanwhile, DNA was adsorbed onto the probe surface at low $\mathrm{pH}$ by direct electrostatic attraction.

Kinetic Analysis of DNA Adsorption onto the Silica Surface. Before the discussion of the DNA adsorption kinetics, 
we focus on the total fluorescence signal detected by EWAB, which is composed of the following two components

$$
I_{\mathrm{t}}=I_{0}+I_{\mathrm{h}}
$$

where $I_{\mathrm{t}}$ is the total fluorescence intensity, $I_{0}$ is the background baseline intensity, and $I_{\mathrm{h}}$ is the contribution from ssDNA adsorption onto the surface. As above mentioned, the contribution of the fluorescence signal from free ssDNA in solution could be negligible. Given that the fluorescence signal (I) is proportional to the coverage $\Gamma$, the eqs $5-7$ can be changed as following

Langmuir adsorption

$$
\frac{I(t)}{I_{\max }}=1-\mathrm{e}^{-k_{\mathrm{a}} C_{0} t / I_{\max }}
$$

Langmuir adsorption with first-order

$$
\frac{I(t)}{I_{\max }}=\frac{K_{\mathrm{A}} C_{0}}{1+K_{\mathrm{A}} C_{0}}\left(1-\mathrm{e}^{-\left(k_{\mathrm{on}} C_{0}+k_{\text {off }}\right) t}\right)
$$

Second-order Langmuir adsorption

$$
\frac{I(t)}{I_{\max }}=\frac{k_{\mathrm{a}} C_{0} t}{I_{\max }+k_{\mathrm{a}} C_{0} t}
$$

where $I_{\max }$ is the maximum fluorescence signal when the DNA probe of the sensor surface is saturated by a target DNA sequence.

To simplify, we reduced the background baseline intensity $\left(I_{0}\right)$ of the real-time kinetic curves, and the initial time was calculated from the sampling. Figure 5 revealed that a part of the ssDNA adsorption kinetic curves determined by EWAB was fitted to various Langmuir models. The fitted Langmuir models depend on the correlation coefficient $\left(R^{2}\right.$ value), which indicates the relationship between the adsorption data and the theoretical models. The parameter $R^{2}$ calculated from various Langmuir models are listed in Table S1. The secondorder Langmuir $R^{2}$ values for the hydroxyl probe, amino probe, and OVA-modified probe in monovalent ion solution were $0.9949,0.9976$, and 0.9971, respectively. These values were higher than those fitted by the other two models. Similarly, the Langmuir $R^{2}$ values for hydroxyl probe, amino probe, and OVA-modified probe in divalent ion solution were 0.9969, 0.9955 , and 0.9970 , respectively. These values were also higher than those fitted by the other two models. These findings signified that the second-order Langmuir sorption model provides better fitting than the Langmuir model and Langmuir model with first-order desorption to describe the DNA adsorption onto the silica samples. Hence, the DNA adsorption was homogeneously applied across the probe surface. To further validate this notion, we employed the Freundlich model to fit the adsorption kinetics model of ssDNA (Figure S4), which was the multilayer adsorption model. The Freundlich $R^{2}$ values were much lower (Table S2), indicating that the Freundlich model did not fit the adsorption kinetic curves of ssDNA. These results suggested that DNA adsorption onto these surfaces was achieved by monolayer adsorption.

\section{DISCUSSION AND CONCLUSIONS}

One chief conclusion from our work is the close relation of the mechanism of DNA adsorption onto solid surfaces with the surface's functional group. Despite the breadth of prior research, the driving force and binding mechanism of DNA adsorption onto the solid surface remain relatively controver- sial. The hydrophobic interactions and hydrogen bonding of unpaired bases were regarded as the main driving force of such DNA adsorption. ${ }^{4}$ Several studies concluded that the phosphate-silanol interaction is an important factor in the interaction between DNA and various types of silica surfaces. ${ }^{10}$ Balladur et al. found that electrostatic interactions between the negatively charged DNA and the amino silica wafers played a major role in the adsorption process. ${ }^{25}$ However, Melzak et al. assumed that intermolecular hydrogen bond formation in the DNA-silica contact layer was responsible for the DNA adsorption. ${ }^{14}$ These controversial conclusions may be contributed to the following reasons. On one hand, the different solid surface pretreatment methods formed various functional surfaces and generated various adsorption mechanisms. On the other hand, most previous studies were performed by bulk depletion methods. Under such strategy, some important dynamic information on the DNA adsorption process is ignored or undetectable and may achieve misleading results.

In our study, real-time kinetic detection provides highly intuitive understanding of DNA adsorption onto the solid surface. Actually, the driving force and binding mechanism of DNA adsorption differ among various functional solid surfaces. First, the adsorption kinetics of DNA is closely related to the hydrophobilic/hydrophobic properties of the solid surface. A bare fiber optic probe is highly hydrophobic because it exists mainly in the bridging ethereal oxygen conformation and possesses no free surface hydroxyl groups. ${ }^{25}$ In this case, very small DNA amounts are adsorbed onto the surface at any salt concentration and $\mathrm{pH}$ condition. However, after the solid surface was modified by hydroxyl and amino groups and protein, the hydrophobic property of the solid surface obviously increased, and adsorption amount of DNA changed with variations in salt concentration and $\mathrm{pH}$. Second, the main driving force of DNA adsorption onto hydroxyl- and proteinmodified solid surfaces originate from the hydrophobic interactions, hydrogen bonding, and the interaction between DNA phosphate and functional groups on the silica surface. The electrostatic repulsion stems from the negative charges of DNA and the silica surface, which can be screened by increasing the ionic strength. Finally, the electrostatic attraction between the negative charge of DNA and positive charge of the amino silica surface is likely the most important factor in DNA adsorption onto the amino solid surface. This electrostatic attraction can be reduced by raising the ionic strength.

Another main result is discrepancy in the effects of monovalent and divalent cation on DNA adsorption. Similar to previous studies, low-ionic-strength divalent cations provide greater attachment efficiencies than high-ionic-strength solutions containing monovalent cations. The balance of ssDNA adsorption onto the hydroxyl- and OVA-modified silica surface is achieved faster in the presence of $\mathrm{Na}^{+}$than in the presence of $\mathrm{Mg}^{2+}$. This difference may be related to the adsorption mechanism of DNA in the presence of $\mathrm{Na}^{+}$or $\mathrm{Mg}^{2+}$. The phosphate diesters on the DNA backbone are strong acids and render DNA a strong polyelectrolyte carrying negative charges at most solution $\mathrm{pH}$. Although monovalent cations (e.g., $\mathrm{Na}^{+}$) do not directly interact with the DNA backbone, these cations tend to shield and stabilize the negatively charged phosphate groups by forming weak ionic or electrostatic bonds. In our experimental results, when a certain monovalent cation is present, the DNA can be effectively neutralized or even converted from negative to positive. This occurrence results in increased DNA adsorption onto the negative surface (e.g., 
hydroxyl- and OVA-modified surfaces) and decreased DNA adsorption onto the amino surface. Moreover, a small amount of DNA adsorbs onto the amino surface in the presence of high $\mathrm{Na}^{+}$concentration. Meanwhile, the divalent electrolyte can compensate for the large negative surface charge density of DNA. $\mathrm{Mg}^{2+}$, which is usually associated with water molecules, tend to link adjacent anions and generally neutralize the negatively charged DNA surface by counterion condensation instead of binding to specific sites. ${ }^{29}$ The dynamic nature of counterion condensation results in the partial occupancy of specific sites at regions of high electrostatic potential, with the level of occupancy being a function of ionic strength. Divalent cations, however, form both indirect and/or direct covalent bonds with either the negatively charged DNA backbone or silanol groups present on the silica scaffold surface. ${ }^{29}$ These points explain why the divalent cations were more effective in driving DNA adsorption than monovalent cations. The kinetics of DNA adsorption onto the amino surface under $\mathrm{Na}^{+}$and $\mathrm{Mg}^{2+}$ were similar. However, the action of $\mathrm{Mg}^{2+}$ was not as effective as that of $\mathrm{Na}^{2+}$ on DNA adsorption onto the amino sensor surface. The part of the DNA remained adsorbed onto the amino surface even at high $\mathrm{Mg}^{2+}$ concentration.

In summary, we presented a quantitative study of ssDNA adsorption onto various functional surfaces. We also discussed mechanism behind this ssDNA adsorption in the presence of monovalent or divalent ions and under various $\mathrm{pH}$ conditions. The mechanisms of ssDNA adsorption onto solid surfaces were greatly dependent on the solid surface properties and the solution characteristics (e.g., ionic strength and $\mathrm{pH}$ ). The DNA adsorption onto various functional surfaces can be perfectly fitted by second-order Langmuir models, indicating that the process is a single-molecular-layer adsorption. The results helped increase understanding on the real-time processes involved in DNA adsorption onto silica and can facilitate the future optimization of biosensors, DNA purification, and genetic analysis and delivery.

\section{EXPERIMENTS}

Chemicals and Materials. OVA, APTS, glutaraldehyde, and sodium dodecyl sulfate were purchased from Sigma-Aldrich (St. Louis, MO). All other reagents, unless otherwise specified were purchased from the Beijing Chemical Agents Co. Ltd. (Beijing, China).

The fluorescence-labeled high-performance liquid chromatography-grade oligonucleotide was purchased from Sangon Biotechnology Co. Ltd. (Shanghai, China). The random sequence of DNA was: 5'-Cy5.5-AGTCA ACTTGA AATGT CCGAT GCTC-3'. DNA oligonucleotide was dissolved in ultrapure water $(\mathrm{pH} 7.0)$ and kept frozen at $-20{ }^{\circ} \mathrm{C}$ for storage.

Surface Functionalization of Optic Fiber Probes. The probes consist of a $5 \mathrm{~cm}$ length of $600 \mu \mathrm{m}$ diameter, plastic-clad step-index quartz optical fiber (Chunhui Science \& Technology Industrial Co., China) with cladding removed from $3.0 \mathrm{~cm}$ along the distal end to form the sensing region (Figure S1). First, this region is tapered using immersion into hydrofluoric acid based on tube etching. ${ }^{21}$ The length of the tapered section and the sensing region are about 0.3 and $3.0 \mathrm{~cm}(\Phi 225 \mu \mathrm{m})$, respectively. The etched optic fiber probe was washed by ultrapure water and regarded as fiber A. Second, the etched probe was placed in piranha solution (concentrated $\mathrm{H}_{2} \mathrm{SO}_{4}$ / $\mathrm{H}_{2} \mathrm{O}_{2} 2: 1$ ) to hydrolyze the probe surface, which increased the silanol group concentration of the probe surface. After acid treatment, the probe was rinsed with ultrapure water and regarded as fiber B. Third, the hydroxyl probe was placed in $2 \%$ APTS in toluene to obtain the amino surface for $1 \mathrm{~h}$ after drying in $\mathrm{N}_{2}$. Excess APTS was eliminated with dry toluene to assure the order and uniformity of the self-assembled monolayer. $^{22}$ After washing by ultrapure water, the amino probe was regarded as fiber $\mathrm{C}$. Next, the amino probe was immersed in a $5.0 \%(\mathrm{v} / \mathrm{v})$ glutaraldehyde solution for $1 \mathrm{~h}$ at 37 ${ }^{\circ} \mathrm{C}$ and completely washed with ultrapure water. Finally, OVA was covalently coupled to the probe surface through the optic fiber probe and was immersed overnight in $2 \mathrm{mg} / \mathrm{mL}$ OVA in PBS solution $\left(\mathrm{pH} \mathrm{7.4)}\right.$ at $4{ }^{\circ} \mathrm{C}$, which was regarded as fiber $\mathrm{D}$ after washing with ultrapure water.

Instrument: EWAB. The EWAB, which was previously developed by our group, was applied to detect the real-time fluorescence signal (Figure S1). ${ }^{21}$

Adsorption of ssDNA Detected by EWAB. In each sample detection process, $300 \mu \mathrm{L}$ of $20 \mathrm{nM}$ Cy5.5-labeled ssDNA was delivered into the sample cell by a peristaltic pump. The ssDNA adsorbed the functional optic fiber probes for a certain time at room temperature, and the fluorescence signal was detected in real time by EWAB.

To investigate effects of ionic species and strength, the DNA was prepared using ultrapure water with various concentrations of $\mathrm{NaCl}(0,0.1,0.25,0.5,1.0$, and $2.0 \mathrm{M})$ or $\mathrm{MgCl}_{2}(0,1.0,5.0$, $10.0,50.0$, and $100.0 \mathrm{mM})$, respectively. To study the effect of $\mathrm{pH}$, the DNA solution was prepared using $0.01 \mathrm{M}$ PBS with different $\mathrm{pH}(\mathrm{pH}=1,3,5,7,9)$, which were adjusted by $1 \mathrm{M}$ $\mathrm{KOH}$ or concentrated $\mathrm{HCl}$.

Analysis of ssDNA Adsorption Kinetics Data. In theory, each existing kinetic model representing adsorption kinetics at solid/liquid interfaces yields a characteristic unique adsorption curve. ${ }^{23}$ However, various kinetic models are practically difficult to test, and reliable kinetic parameters are challenging to extract. Real-time data are needed over a wide range of surface adsorption coverage and time scale. Despite the emergence of a number of powerful surface analytical techniques, quantitation of absolute surface adsorption coverage at solid/solution interfaces remains a challenge. In this section, the resulting time-dependent surface coverage $\Gamma$ was compared with various Langmuir adsorption models as follows:

Langmuir adsorption

$$
\frac{\mathrm{d} \Gamma(t)}{\mathrm{d} t}=k_{\mathrm{a}} C_{0}\left(1-\frac{\Gamma(t)}{\Gamma_{\max }}\right)
$$

Langmuir adsorption with first-order desorption

$$
\frac{\mathrm{d} \Gamma(t)}{\mathrm{d} t}=k_{\mathrm{a}} C_{0}\left(1-\frac{\Gamma(t)}{\Gamma_{\max }}\right)-k_{\mathrm{d}} \Gamma(t)
$$

Second-order Langmuir adsorption

$$
\frac{\mathrm{d} \Gamma(t)}{\mathrm{d} t}=k_{\mathrm{a}} C_{0}\left(1-\frac{\Gamma(t)}{\Gamma_{\max }}\right)^{2}
$$

where $C_{0}$ is the ssDNA initial concentration, $k_{a}$ is the adsorption constant, $k_{\mathrm{d}}$ is the desorption constant, and $\Gamma_{\max }$ is the maximum surface coverage. The Langmuir model assumes that all binding sites are equivalent and already occupied sites do not influence the binding reaction in adjacent places. The model also assumes that the surface is homogenously covered by monolayers. ${ }^{24}$ 


\section{ASSOCIATED CONTENT}

\section{S Supporting Information}

The Supporting Information is available free of charge on the ACS Publications website at DOI: 10.1021/acsomega.7b02063.

Scheme of the EWAB; results of XPS; adsorption kinetic curves; correlation coefficient from Langmuir and Freundlich models; and the contact angle (PDF)

\section{AUTHOR INFORMATION}

\section{Corresponding Authors}

*E-mail: Chuanna0306@163.com (A.N.Z.).

*E-mail: Longf04@ruc.edu.cn (F.L.).

ORCID

Feng Long: 0000-0002-3372-0624

Notes

The authors declare no competing financial interest.

\section{ACKNOWLEDGMENTS}

This research was financially supported by the National Natural Science Foundation of China (NSF) funded by the Chinese government $(21277173,21675171)$, the National Instrument Major Project of China, funded by Ministry of Science and Technology of the People's Republic of China (2012YQ3011105), and the Fundamental Research Funds for the Central Universities from Renmin University of China funded by the Chinese government (15XNLD04).

\section{REFERENCES}

(1) Fodor, S. P. A.; Rava, R. P.; Huang, X. C.; Pease, A. C.; Holmes, C. P.; Adams, C. L. Multiplexed biochemical assays with biological chips. Nature 1993, 364, 555-556.

(2) Rittich, B.; Španova, A. SPE and purification of DNA using magnetic particles. J. Sep. Sci. 2013, 36, 2472-2485.

(3) Lockhart, D. J.; Winzeler, E. A. Genomics, gene expression and DNA arrays. Nature 2000, 405, 827-836.

(4) Hikosaka, R.; Nagata, F.; Tomita, M.; Kato, K. Adsorption and desorption characteristics of DNA onto the surface of amino functional mesoporous silica with various particle morphologies. Colloids Surf., B 2016, 140, 262-268.

(5) Torney, F.; Trewyn, B. G.; Lin, V. S.-Y.; Wang, K. Mesoporous silica nanoparticles deliver DNA and chemicals into plants. Nat. Nanotechnol. 2007, 2, 295-300.

(6) Xia, T. A.; Kovochich, M.; Liong, M.; Meng, H.; Kabehie, S.; George, S.; Zink, J. I.; Nel, A. E. Polyethyleneimine coating enhances the cellular uptake of mesoporous silica nanoparticles and allows safe delivery of siRNA and DNA constructs. ACS Nano 2009, 3, 32733286.

(7) Xiao, Y.; Lubin, A. A.; Heeger, A. J.; Plaxco, K. W. Label-free electronic detection of thrombin in blood serum by using an aptamerbased sensor. Angew. Chem., Int. Ed. 2005, 44, 5456-5459.

(8) Pedano, M. L.; Rivas, G. A. Immobilization of DNA on glassy carbon electrodes for the development of affinity biosensors. Biosens. Bioelectron. 2003, 18, 269-277.

(9) Dabrowski, J.; Müssig, H. J. Silicon Surfaces and Formation of Interfaces: Basic Science in the Industrial World; World Scientific: River Edge, NJ, 2000.

(10) Shi, B.; Shin, Y. K.; Hassanali, A. A.; Singer, S. J. DNA Binding to the Silica Surface. J. Phys. Chem. B 2015, 119, 11030-11040.

(11) Kan, Y.; Tan, Q.; Wu, G.; Si, W.; Chen, Y. Study of DNA adsorption on mica surfaces using a surface force apparatus. Sci. Rep. 2015, 5, 8442 .

(12) Vandeventer, P. E.; Lin, J. S.; Zwang, T. J.; Nadim, A.; Johal, M. S.; Niemz, A. Multiphasic DNA Adsorption to Silica Surfaces under Varying Buffer, pH, and Ionic Strength Conditions. J. Phys. Chem. B 2012, 116, 5661-5670.
(13) Kang, S. H.; Shortreed, M. R.; Yeung, E. S. Real-time dynamics of single-DNA molecules undergoing adsorption and desorption at liquid-solid interfaces. Anal. Chem. 2001, 73, 1091-1099.

(14) Melzak, K. A.; Sherwood, C. S.; Turner, R. F. B.; Haynes, C. A. Driving forces for DNA adsorption to silica in perchlorate solutions. J. Colloid Interface Sci. 1996, 181, 635-644.

(15) Berney, H. B.; Oliver, K. Dual polarization interferometry size and density characterisation of DNA immobilisation and hybridisation. Biosens. Bioelectron. 2005, 21, 618-626.

(16) Scholes, C. A.; Millar, D. P.; Gee, M. L.; Smith, T. A. Resonance Energy-Transfer Studies of the Conformational Change on the Adsorption of Oligonucleotides to a Silica Interface. J. Phys. Chem. B 2011, 115, 6329-6339.

(17) Kang, S. H.; Shortreed, M. R.; Yeung, E. S. Real-time dynamics of single-DNA molecules undergoing adsorption and desorption at liquid-solid interfaces. Anal. Chem. 2001, 73, 1091-1099.

(18) Isailovic, S.; Li, H.-W.; Yeung, E. S. Adsorption of single DNA molecules at the water/fused-silica interface. J. Chromatogr. A 2007, $1150,259-266$.

(19) Lillis, B.; Manning, M.; Berney, H.; Hurley, E.; Mathewson, A.; Sheehan, M. M. Dual polarisation interferometry characterisation of DNA immobilisation and hybridisation detection on a silanised support. Biosens. Bioelectron. 2006, 21, 1459-1467.

(20) Kato, N.; Lee, L.; Chandrawati, R.; Johnston, A. P. R.; Caruso, F. Optically Characterized DNA Multilayered Assemblies and Phenomenological Modeling of Layer-by-Layer Hybridization. J. Phys. Chem. C 2009, 113, 21185-21195.

(21) Long, F.; Shi, H. C.; He, M.; Zhu, A. N. Sensitive and rapid detection of 2,4-dicholoro phenoxyacetic acid in water samples by using evanescent wave all-fiber immunosensor. Biosens. Bioelectron. 2008, 23, 1361-1366.

(22) Bhatia, S. K.; Shrive-Lake, L. C.; Prior, K. J.; Georger, J. H.; Calvert, J. M.; Bredehorst, R.; Ligler, F. S. Use of thiol-terminal silanes and heterobifunctional crosslinkers for immobilization of antibodies on silica surfaces. Anal. Biochem. 1989, 178, 408-413.

(23) Peterson, A. W.; Heaton, R. J.; Georgiadis, R. Kinetic Control of Hybridization in Surface Immobilized DNA Monolayer Films. J. Am. Chem. Soc. 2000, 122, 7837-7838.

(24) Fisher, R. J.; Fivash, M.; Casas-Finet, J.; Erickson, J. W.; Kondoh, A.; Bladen, S. V.; Fisher, C.; Watson, D. K.; Papas, T. Realtime DNA binding measurements of the ETS1 recombinant oncoproteins reveal significant kinetic differences between the p42 and p51 isoforms. Protein Sci. 2008, 3, 257-266.

(25) Balladur, V.; Theretz, A.; Mandrand, B. Determination of the Main Forces Driving DNA Oligonucleotide Adsorption onto Aminated Silica Wafers. J. Colloid Interface Sci. 1997, 194, 408-418.

(26) Parida, S. K.; Dash, S.; Patel, S.; Mishra, B. K. Adsorption of organic molecules on silica surface. J. Colloid Interface Sci. 2006, 121, $77-110$.

(27) Nguyen, T. H.; Chen, K. L. Role of divalent cations in plasmid DNA adsorption to natural organic matter-coated silica surface. Environ. Sci. Technol. 2007, 41, 5370-5375.

(28) Nguyen, T. H.; Elimelech, M. Adsorption of plasmid DNA to a natural organic matter-coated silica surface: Kinetics, conformation, and reversibility. Langmuir 2007, 23, 3273-3279.

(29) Huang, F.; Liang, H. Adsorption Behaviors of DNA/Cation Complexes on Amino and Silica Chip Surfaces: A Dual Polarization Interferometry Study. ACS Appl. Mater. Interfaces 2013, 5, 5025-5033.

(30) Matsuura, Y.; Arakawa, S.; Okamoto, M. Single-stranded DNA adsorption characteristics by hollow spherule allophane nano-particles: $\mathrm{pH}$ dependence and computer simulation. Appl. Clay Sci. 2014, 101 , 591-597. 